\title{
Effect of raising body temperature on visual and somatosensory evoked potentials in patients with multiple sclerosis
}

\author{
W. B. M A T THEWS, D. J.REA D, A N D ER IKA POUNT NEY \\ From the Department of Clinical Neurology, University of Oxford, The Churchill Hospital, Oxford
}

SUMMARY The effects of raising body temperature on the visual (VEP) and somatosensory (SEP) evoked potentials were observed in normal subjects and in patients with multiple sclerosis. The amplitude of the VEP was significantly reduced to the same degree after heating in normal subjects and in patients with multiple sclerosis but there was no effect on the latency of the potential. Changes in amplitude could not be related to reduction in acuity. In contrast, the cervical SEP was greatly disorganised after heating in many patients with multiple sclerosis while the only effect in normal subjects was to reduce the latency by increasing peripheral conduction velocity. These results suggest that heat caused conduction block in demyelinated axons in the sensory pathways of the cervical spinal cord.

Many patients with multiple sclerosis experience profound exacerbation of their symptoms after a hot bath, and this sensitivity to heat may be so extreme that even a hot drink will cause blurred vision. The effect of heat on myelinated fibres is to increase conduction velocity up to a critical temperature when conduction block develops abruptly (Schauf and Davis, 1974). In demyelinated fibres the increase in velocity is less and blocking occurs at a much lower temperature (Rasminsky, 1973). The natural assumption is, therefore, that the temporary adverse effect of heat in patients with multiple sclerosis is caused by conduction block in demyelinated fibres.

Averaging techniques have allowed the use of visual (VEP), auditory (AEP), and somatosensory (SEP) evoked potentials in the diagnosis of multiple sclerosis (Halliday et al., 1973; Robinson and Rudge, 1977; Small et al., 1978). Prolonged latency, particularly of VEP, has been attributed to slowing of conduction within the central nervous system while reduction in amplitude has been related to the number of axons in which conduction is blocked (Halliday and McDonald, 1977). The validity of all these assumptions can be examined,

Address for reprint requests: Professor W. B. Matthews, Department of Clinical Neurology, The Churchill Hospital, Headington, Oxford OX3 $7 \mathrm{LJ}$.

Accepted 2 October 1978 at least in part, by observing the effect of raising body temperature on the evoked potentials.

\section{Methods}

Pattern reversal VEPs were recorded by the methods described by Matthews et al. (1977), the upper limit of the normal value for latency of P100 wave being $111 \mathrm{~ms}$ (mean $+2.5 \mathrm{SD})$. Although multichannel recordings were made, the only one referred to in this communication is that derived from an electrode $50 \mathrm{~mm}$ above the inion to a frontal reference electrode.

Cervical and short latency cortical evoked potentials were recorded by the method described by Small et al. (1978). An additional electrode was placed above the clavicle in proximity to the brachial plexus and referred to the scalp electrode Fz.

In 14 patients with multiple sclerosis, 11 in the definite and three in the probable categories of McAlpine (1972), VEP and SEP were recorded before and after oral temperature had been raised by approximately $1^{\circ} \mathrm{C}$ by exposure of the trunk to a heat cradle. In two of these patients similar recordings were made before and after raising oral temperature by $0.5^{\circ} \mathrm{C}$ in a hot room. This latter method of heating was also used in one patient with clinical evidence of isolated optic nerve in- 
volvement, VEP only being recorded. Six normal subjects were examined in the same way before and after raising oral temperature by $1^{\circ} \mathrm{C}$. In two of the patients with multiple sclerosis and two of the normal subjects recordings were also made after an interval of one hour without heating.

\section{Results}

\section{VISUAL EVOKED POTENTIALS}

In normal subjects heating did not affect visual acuity. There was no significant change in latency of $\mathrm{P} 100$ wave $(\mathrm{N}=12$; mean change $-0.8 \mathrm{~ms}$; range $-3-+3 \mathrm{~ms})$. The mean amplitude of $\mathrm{P} 100$ before heating was $8.3 \mu \mathrm{V}$ and after heating $6.9 \mu \mathrm{V}$, reduction occurring in the response from 10 eyes. This reduction was statistically significant $(\mathrm{N}=12$; mean change $-1.4 \pm \mathrm{S}$.D. $1.3 \mu \mathrm{V} ; \mathrm{P}<0.01$, Wilcoxon).

In the 17 experiments on 15 subjects with multiple sclerosis visual acuity was reduced after heating, usually by one line on the Snellen chart at $6 \mathrm{~m}$, in 14 eyes in eight patients. Acuity did not change in the remaining 20 examinations which included the two patients who were heated twice.

Latency of the P100 could be measured before heating in 27 eyes in patients with multiple sclerosis, and in 20 of these it was abnormally prolonged, including all those where acuity was re- duced after heating. There was no significant change in latency after heating (mean change $-1.3 \mathrm{~ms}$ ), with the exception of one patient in whom latency was greatly reduced from both eyes. In the seven eyes where initial latency was normal the mean change of $2.0 \mathrm{~ms}$ was again not significant, but in one of these patients examined twice the increase was sufficient to render the response abnormal. In the seven instances where the VEP could not be measured initially there was no change in five but in one patient a measurable potential was obtained from each eye after heating.

In patients with multiple sclerosis in the 27 instances where measurement was possible the mean amplitude of P100 before heating was $6.6 \mu \mathrm{V}$ and after heating $5.0 \mu \mathrm{V}$, reduction occurring in 23 eyes (Figs. 1 and 2 ). The reduction was statistically significant $(\mathrm{N}=27$; mean change $-1.6 \pm \mathrm{SD}$ $1.7 \mu \mathrm{V} ; \mathrm{P}<0.01$ Wilcoxon). There was no significant difference between the amplitude reduction in patients with multiple sclerosis and in normal subjects, nor did reduction of visual acuity after heating affect the result.

In subjects examined after an interval of one hour without heating no change in vision or in latency or amplitude of VEP occurred.

SOMATOSENSORY EVOKED POTENTIALS

In normal subjects heating caused discomfort but
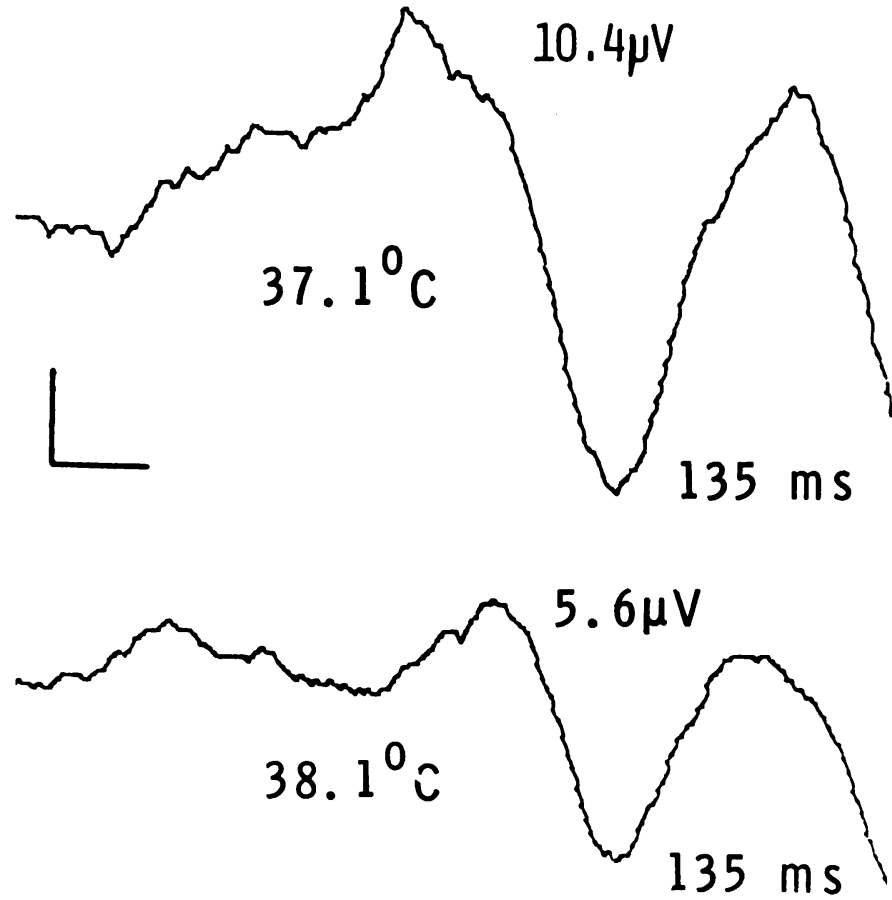

Fig. 1 Visual evoked potential from right eye of patient with multiple sclerosis before and after heating, showing reduction of amplitude with no change in latency. Vertical bar $2 \mu V$; horizontal bar $20 \mathrm{~ms}$. 

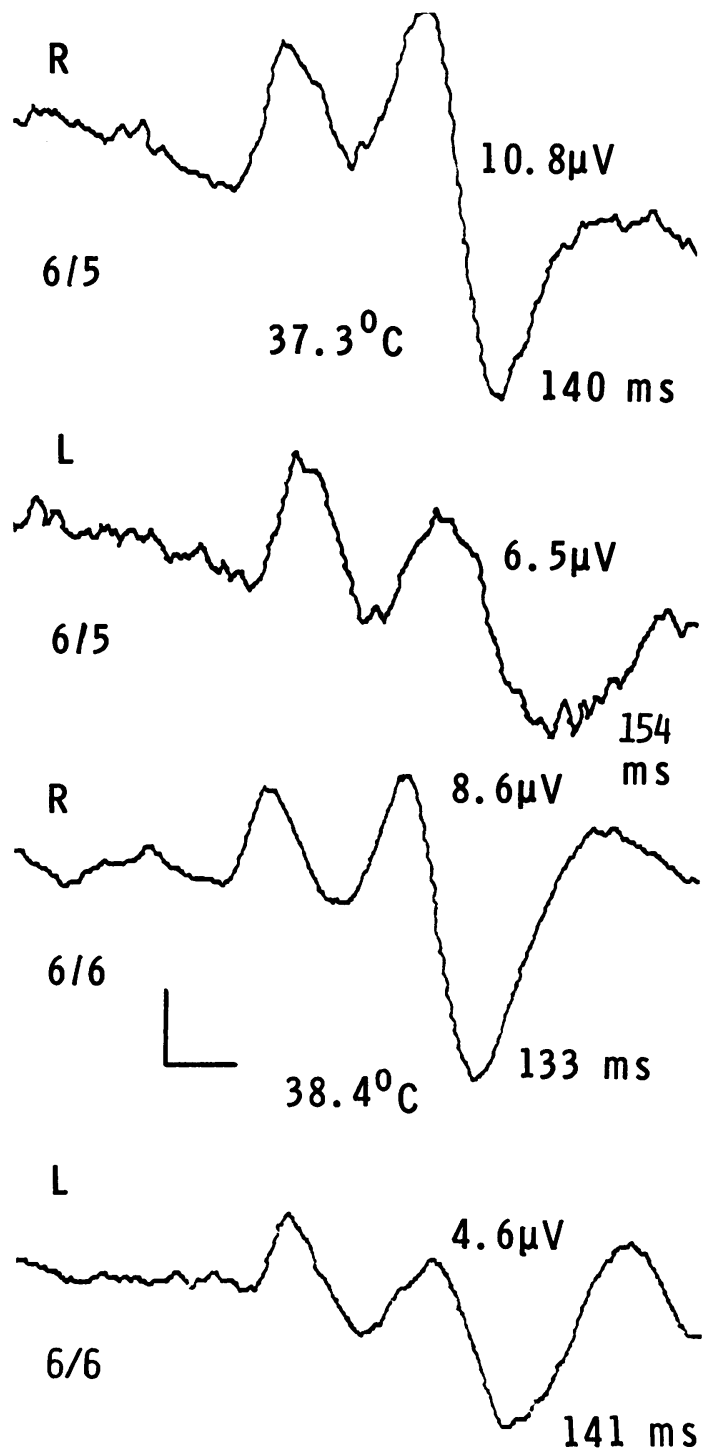

Fig. 2 Visual evoked potential from right and left eyes in a patient with multiple sclerosis, the top two lines before heating and the lower two after raising oral temperature $1.1^{\circ} \mathrm{C}$. Amplitude is reduced and in this case latency is also reduced. Vertical bar $2 \mu \mathrm{V}$; horizontal bar $20 \mathrm{~ms}$.

no other symptoms. The effect on SEP was to shorten the latency of N13 and N20 waves, the former by $0.7 \pm \mathrm{SD} 0.3 \mathrm{~ms}$ and the latter by $1.0 \pm$ SD $0.8 \mathrm{~ms}(\mathrm{~N}=12)$. This was almost entirely caused by increased conduction velocity in the peripheral nervous system, and the difference between the latency of $\mathrm{N} 20$ and that of the potential recorded over the brachial plexus, representing central conduction, was little altered by heat $(\mathrm{N}=$ 12; mean difference $-0.18 \pm \mathrm{SD} 0.6 \mathrm{~ms}$ ). The amplitude of the normal N13 was reduced in seven instances but the mean reduction of $0.5 \pm \mathrm{SD}$ $0.7 \mu \mathrm{V}$ did not reach statistical significance.

In six patients with multiple sclerosis, raising oral temperature by $1{ }^{\circ} \mathrm{C}$ caused a pronounced temporary increase in weakness and ataxia. This had been expected by the patients who were familiar with the effects of a hot bath. In five of these patients there was increased sensory loss in the lower limbs after heating, involving both postural and vibration sense in three and the latter only in two. Cutaneous sensation and sensation in the upper limbs were not disturbed. In a further two patients the only observed effect of heating was the development of nystagmus, and in another patient plantar reflexes became extensor. In four patients with minimal signs of the disease at the time of the experiment there was no change in physical signs. In one patient ataxia became less severe after heating.

Of the 32 cervical SEPs from 14 patients (two being examined twice) 17 were normal before heating. Of the remaining 15 , five were absent, in six the main peak of $\mathrm{N} 13$ was absent, and in four latency of N13 was abnormally prolonged. After heating 12 potentials were normal, this total including two where reduction of abnormal latency to normal values occurred in one patient. Twenty cervical SEPs were abnormal after heating of which 12 potentials were now absent, the abnormalities in the remaining eight examples consisting of prolongation of latency and distortion of the waveform with loss of recognisable N13 peak (Figs. 3 and 4). Seven potentials, normal before heating, had become abnormal. The amplitude of the potential, whether normal or abnormal, was reduced in nearly every instance, but statistical comparison with normal subjects was not possible because of the difficulty of measuring low voltage distorted potentials.

In the six patients in whom pronounced exacerbation of disability followed heating, eight of the 12 cervical SEPs were initially abnormal. After heating 10 were abnormal but abnormally prolonged latency had been reduced to normal in the remaining two potentials. In the four patients with minimal evidence of multiple sclerosis at the time of examination, and in whom heat caused no deterioration, six of the eight cervical SEPs were initially normal and remained normal after heating.

Before heating the latency of N20 was abnormally prolonged in nine instances and one potential 

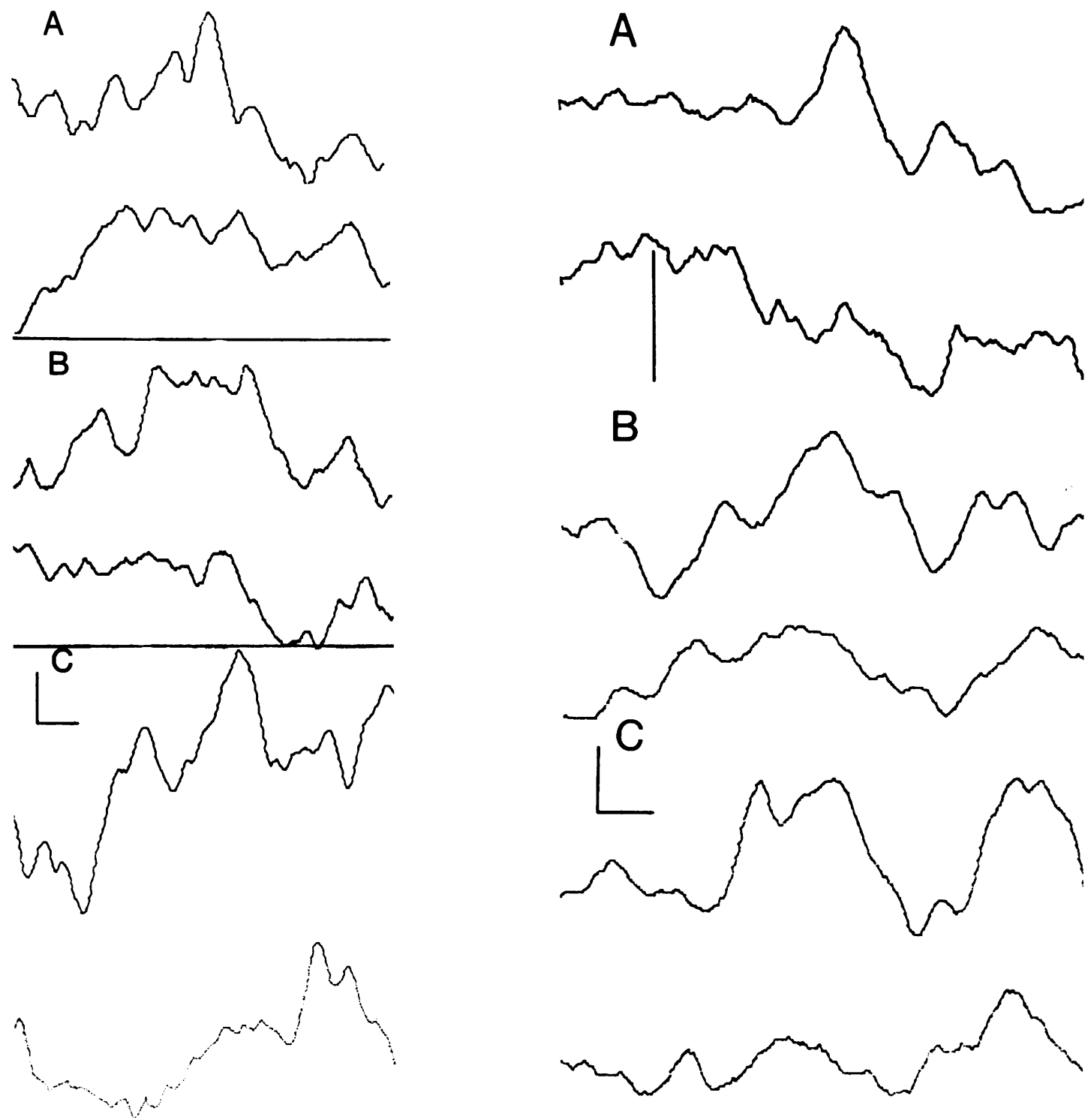

Figs. 3 and 4 Cervical SEP recorded from six patients with multiple sclerosis. In each pair $(A, B$, and $C)$ the upper trace was recorded before heating and the lower after raising oral temperature by $1^{\circ} \mathrm{C}$. The great reduction of amplitude and virtual abolition of the potential can be seen in each instance. Vertical bars $1 \mu V$; horizontal bars $2 \mathrm{~ms}$.

was absent. The effect of heating was to reduce the latency. In the 29 examples where it was possible to measure latency before and after heating the mean reduction was $-1.4 \pm \mathrm{SD} 1.1 \mathrm{~ms}$. Abnormal latency returned to normal values in only two examples.

Evidence was sought for changes in conduction velocity within the central nervous system by measuring the difference between the latency of N20 and that of the potential recorded over the brachial plexus. Before heating the main difference was $11.3 \pm \mathrm{SD} 2.2 \mathrm{~ms}(\mathrm{~N}=24)$ compared with the normal in this series of $9.5 \pm \mathrm{SD} 1.2 \mathrm{~ms}(\mathrm{~N}=$ 12). This result did not reach statistical significance $(P<0.1>0.05$, Student's $t$ test $)$. The mean reduction in the difference between the two latencies 
produced by heat was greater than in the normal subjects $(\mathrm{N}=24$; mean change $-0.8 \pm 1.2 \mathrm{~ms}$, compared with the normal mean change of $-0.18 \pm$ SD $0.6 \mathrm{~ms}$ ) but the difference was not significant $(P<0.1>0.05$, Student's $t$ test $)$.

No significant change was observed in SEP in subjects examined after an interval of one hour without heating.

\section{Discussion}

The results showed that exposure to heat in these experiments reduced the amplitude of VEP both in normal subjects and in patients with multiple sclerosis. The changes were unrelated to any effect on visual acuity. In contrast heat had a severely adverse effect on cervical SEP in multiple sclerosis, normal potentials sometimes becoming abnormal and potentials initially abnormal becoming further disorganised. These changes were more often seen in patients in whom heat induced exacerbation of their symptoms. That they were not caused by faulty electrode contact with the passage of time was shown by the absence of any reduction in amplitude in subjects observed for the same length of time but not heated. If faulty contact had resulted from sweating the cervical potential in particular would have been replaced by noise both in normal subjects and in patients with multiple sclerosis. Heat, therefore, has a different effect on these two forms of evoked potential.

As already mentioned it has been suggested that the latency of VEP provides some indication of conduction velocity in visual pathways while the amplitude may be related to the number of conducting axons in the optic nerve (Halliday and McDonald, 1977). The effect of increasing temperature on demyelinated axons is to produce conduction block rather than slowing of conduction velocity (Schauf and Davis, 1974). If these conclusions are correct the present results indicate that conduction is blocked in some axons in the optic nerve in normal subjects by increasing temperature to the extent effected in these experiments. The decline in acuity in many patients with multiple sclerosis shows that heat exerted an adverse effect on the visual system. If this were caused by conduction block in demyelinated fibres it was not reflected in any change in VEP distinguishable from that observed in normal subjects in whom no change in acuity occurred. Persson and Sachs (1978) found that exercise caused reduction of visual acuity and of amplitude of VEP, without change in latency from the affected eye in two patients with permanent after effects of optic neuritis, the potential from the normal eye not being affected. Oral temperature did not change in one of their patients. More detailed examination of visual function is obviously desirable but reliable charting of visual fields would not have been possible in these patients emerging from an hour under a heat cradle.

The $P 100$ wave is a convenient component of the VEP to measure as it is virtually constant in normal subjects and has a sharp peak. It is not the earliest recordable deflection after visual stimulation nor have the events of the $100 \mathrm{~ms}$ between stimulation and this response been clearly established. Both amplitude and latency must be influenced by the relative synchronicity of the electrophysiological discharges making up the recorded potential, an aspect that is not at present amenable to measurement. Regan et al. (1977) observed in multiple sclerosis patients a lack of correspondence between the effects of heating or cooling on visual perception and on the latency of VEP obtained by the different method of sine wave flicker. The use of VEP in the diagnosis of multiple sclerosis is largely based on this dissociation between abnormalities of function and of the recorded potentials, allowing the recognition of clinically silent plaques.

Short latency SEP recorded from the neck and scalp can be far more closely related to conduction velocity within the central nervous system. The origin of the different components has not been definitely established but evidence suggests that they derive from fixed sites and do not resemble a "travelling" wave such as the compound action potential of peripheral nerve (Matthews et al., 1974). The latencies of $\mathrm{N} 13$ and $\mathrm{N} 20$ are compatible with an origin within the spinal cord or brainstem and the cerebral cortex respectively (Matthews et al., 1974). In normal subjects these potentials were not influenced by an increase in body temperature except for reduction in latency attributable to increased peripheral conduction velocity and a slight reduction in amplitude. The effects in many patients with multiple sclerosis, particularly when N13 was already abnormal, were to disorganise the potential and in particular to reduce its amplitude. In patients in whom heat induced exacerbation of their symptoms the cervical SEP was usually abnormal before heating and further deteriorated during the test, but this effect could equally be related to severity of the disease. The simplest explanation is that increasing temperature did indeed cause conduction block within the sensory pathways of the cervical spinal cord. The short latency SEP are of brief duration and low voltage and could well be more vulnerable to the effects of conduction block in preventing 
the synchronous discharges that normally allow the potential to be recorded. The dissociation between loss of function and abnormality of evoked potential, while still of ten present, was less marked than in the visual system.

\section{References}

Halliday, A. M., and McDonald, W. I. (1977). Pathophysiology of demyelinating disease. British Medical Bulletin, 33, 21-27.

Halliday, A. M., McDonald, W. I., and Mushin, J. (1973). Visual evoked response in diagnosis of multiple sclerosis. British Medical Journal, 4, 661664.

McAlpine, D. (1972). In Multiple Sclerosis: A Reappraisal, p. 202. Edited by D. McAlpine, C. E. Lumsden and E. D. Acheson. Churchill Livingstone: Edinburgh and London.

Matthews, W. B., Beauchamp, M., and Small, D. G. (1974). Cervical somatosensory evoked responses. Nature (London), 252, 230-232.

Matthews, W. B., Small, D. G., Small, M., and Pountney, E. (1977). The pattern reversal evoked visual potential in the diagnosis of multiple sclerosis.
Journal of Neurology, Neurosurgery, and Psychiatry, 40, $1009-1014$.

Persson, H. E., and Sachs, C. (1978). Provoked visual impairment in multiple sclerosis studied by visual evoked responses. Electroencephalography and Clinical Neurophysiology, 44, 664-668.

Rasminsky, M. (1973). The effects of temperature on conduction in demyelinated single nerve fibres. Archives of Neurology (Chicago), 28, 287-292.

Regan, D., Murray, T. J., and Silver, R. (1977). Effect of body temperature on visual evoked potential delay and visual perception in multiple sclerosis. Journal of Neurology, Neurosurgery, and Psychiatry, 40, 1083-1091.

Robinson, K., and Rudge, P. (1977). Abnormalities of the auditory evoked potentials in patients with multiple sclerosis. Brain, 100, $19-40$.

Schauf, C. L., and Davis, F. A. (1974). Impulse conduction in multiple sclerosis: a theoretical basis for modification by temperature and pharmacological agents. Journal of Neurology, Neurosurgery, and Psychiatry, 37, 152-161.

Small, D. G., Matthews, W. B., and Small, M. (1978). The cervical somatosensory evoked potential (SEP) in the diagnosis of multiple sclerosis. Journal of the Neurological Sciences, 35, 211-224. 